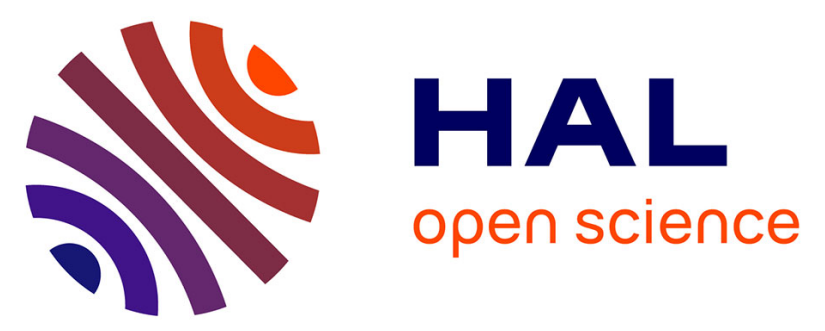

\title{
Time-domain model of the ultrasonic wave propagation in an inhomogeneous anisotropic viscoelastic fluid/solid multilayer medium: application to cortical bone
}

\author{
G. Haïat, Salah Naili, M.-B. Vu, Q. Grimal, M. Talmant, Christophe
}

Desceliers, Christian Soize

\section{To cite this version:}

G. Haïat, Salah Naili, M.-B. Vu, Q. Grimal, M. Talmant, et al.. Time-domain model of the ultrasonic wave propagation in an inhomogeneous anisotropic viscoelastic fluid/solid multilayer medium: application to cortical bone. 2009 IEEE International Ultrasonics Symposium, Sep 2009, Rome, Italy.

pp.Pages: 1-4. hal-00692825

\section{HAL Id: hal-00692825 \\ https://hal.science/hal-00692825}

Submitted on 1 May 2012

HAL is a multi-disciplinary open access archive for the deposit and dissemination of scientific research documents, whether they are published or not. The documents may come from teaching and research institutions in France or abroad, or from public or private research centers.
L'archive ouverte pluridisciplinaire HAL, est destinée au dépôt et à la diffusion de documents scientifiques de niveau recherche, publiés ou non, émanant des établissements d'enseignement et de recherche français ou étrangers, des laboratoires publics ou privés. 


\section{Time-domain model of the ultrasonic wave propagation in an inhomogeneous anisotropic viscoelastic fluid/solid multilayer medium: application to cortical bone}

\author{
G. Haïat ${ }^{1}$, S. Naili ${ }^{2}$, M.-B. Vu ${ }^{2}$, Q. Grimal ${ }^{3}$, M. \\ Talmant ${ }^{3}$, C. Desceliers ${ }^{4}$, C. Soize ${ }^{4}$ \\ ${ }^{1}$ CNRS, UMR CNRS 7052 B2OA, Université Paris 7, \\ 75010 Paris, France \\ ${ }^{2}$ Université Paris 12 - Val de Marne, Laboratoire de \\ Mécanique Physique, 94010 Créteil, France
}

\author{
${ }^{3}$ Université Pierre et Marie Curie-Paris6, Laboratoire
} d'Imagerie Paramétrique, Paris, F-75005 France; CNRS, LIP, Paris, F-75006 France

${ }^{4}$ Université Paris-Est, Laboratoire de Modélisation et Simulation Multi-Echelle, MSME FRE3160 CNRS, 77454 Marne la Vallée, France

guillaume.haiat@univ-paris-diderot.fr

\begin{abstract}
Cortical bone quality is assessed in clinical practice using axial transmission (AT) devices, which allow the measurement of quantitative ultrasonic parameters such as the first arriving signal (FAS) velocity. However, the physical interaction between an ultrasonic wave and cortical bone remains poorly understood due to the complex nature of the bone structure. Cortical bone and the surrounding soft tissues are attenuating media, which might affect the analysis of the results obtained with AT devices. Moreover, cortical bone is highly heterogeneous and a gradient of material properties from the outer to inner part of the cortical shell has been reported. The aim of this work is to evaluate the effect of anisotropic heterogeneous dissipative phenomena occurring in bone and in soft tissues on the ultrasonic response of the bone structure.
\end{abstract}

A two-dimensional finite element time-domain method is derived to model transient wave propagation in a three-layer medium composed of an inhomogeneous transverse isotropic viscoelastic solid layer sandwiched between two dissipative acoustic fluid layers. The model couples acoustic propagation in both dissipative acoustic fluid media with the response of the solid whose constitutive equation is based on the linear theory of viscoelasticity without memory. Bone viscoelasticity is assumed to be heterogeneous and a constant spatial gradient of viscoelastic properties is considered for a value of bone thickness corresponding to a relatively thick bone.

Realistic variations of the viscosity of the soft tissues within the physiological range do not affect the FAS velocity in any configuration. However, when the viscoelastic bone parameters vary within their physiological range, changes of the value of the FAS velocity (up to $301 \mathrm{~m} / \mathrm{s}$ ) are comparable to what has been obtained for variation of the elastic parameters [1]. The components of the viscoelastic tensor affecting the results are the same as those of the stiffness tensor found in [1].

Our results highlight the importance of accounting for absorption phenomena occurring in cortical bone for the analysis of ultrasonic measurements with AT device.
Viscoelasticity, viscosity, quantitative ultrasonic techniques (QUS), finite element method, axial transmission technique, cortical bone. (key words)

\section{INTRODUCTION}

Different metabolic diseases such as osteoporosis may affect bone quality, resulting in a decrease of bone mass and micro-architectural deterioration of bone tissue. In axial transmission (AT) technique (which is particularly adapted to cortical long bone evaluation), both ultrasound emitter and receivers are placed in the same side of the investigated skeletal site. The earliest event or wavelet (usually called First Arriving Signal, FAS) of the multicomponent signal recorded by the receivers has been the most widely investigated. The wave velocity associated with this signal, which is measured in the time domain, can be used to discriminate healthy subjects from osteoporotic patients and is therefore considered as a relevant index of bone status [2,3]. Both experimental [4] and simulation studies [5] have shown that the FAS velocity was related to different bone properties. Numerical simulations have been employed to show that when the cortical thickness is comparable or larger than the wavelength, the type of wave contributing to the FAS corresponds to a lateral wave, whereas when the wavelength is larger than the cortical thickness divided by four, the received signal corresponding to the FAS comes from the first symmetric Lamb wave mode $S_{0}$ guided by the cortical thickness [6].

From a biomechanical point of view, cortical bone is a complex transverse isotropic medium. At the macroscopic scale, porosity in the radial direction (which is associated with the cross-section of the bone) is heterogeneous at all ages and for both genders [7, 8]: the mean porosity in inner part of the bone is significantly higher than in the outer part of the bone. Moreover, cortical bone is a strongly attenuating medium where ultrasonic propagation occurs with losses [9, 10]. Interestingly, Broadband Ultrasonic Attenuation (BUA, defined as the slope of the curve of the frequency dependent attenuation coefficient) measurements performed by the group 
of some of the authors have recently been shown to be significantly related to the microstructure as well as to bone physical properties such as mass density and bone mineral density [10].

The potential advantage of numerical simulation tools over experimental approaches is that it can be used to determine the influence of each bone property independently. Recently, our group has determined the effect of heterogeneous material bone properties on the the FAS velocity using 2-D finite element model (FEM) [1]. Most models of AT developed in the past have considered cortical bone and the surrounding soft tissues as elastic materials. However, the influence of ultrasonic attenuation on the ultrasonic response of the investigated anatomical site in the framework of the AT device remains unclear. Studying the influence of attenuation in bone on the FAS velocity is of particular interest since BUA has been suggested as a potential indicator of bone status.

The aim of this paper is to assess the effect of the viscoelastic nature of cortical bone and of the viscous nature of the surrounding soft tissues on the ultrasonic response obtained with an AT device. Here, bone is modeled as an anisotropic (transverse isotropic) heterogeneous (a gradient of material properties in the radial direction is considered) viscoelastic material and the surrounding soft tissues are modeled as homogeneous viscous liquids.

\section{METHOD}

The approach employed to determine the influence of absorption is described below and more details can be found in [11].

\section{A. Axial transmission configuration}

The geometrical configuration used in the present study is the same as the one used in [1]. Briefly, cortical bone is modeled as a two-dimensional multilayer medium composed of one heterogeneous viscoelastic transverse isotropic solid layer (corresponding to cortical bone) sandwiched between two homogeneous viscous fluid layers as shown in Fig. 1.

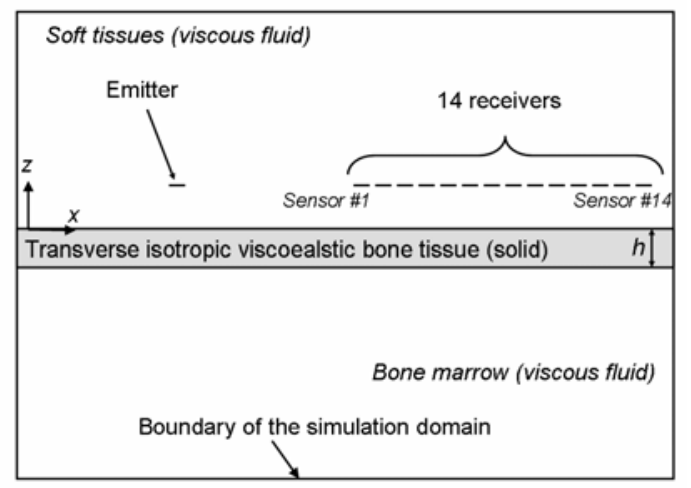

Figure 1. Schematic representation of the simulation domain corresponding to a three-layer medium. The emitter and receivers are indicated by indents.

In the simulation, a pressure source is positioned in the fluid and the excitation signal is a Gaussian pulse with a center frequency of $1 \mathrm{MHz}$. The geometrical arrangement mimics that of an actual probe developed by the 'Laboratoire d'Imagerie Paramétrique' (France) [4]. The FAS velocity estimated is given by the slope of the position of each sensor versus time delays, obtained through a least-square linear regression.

\section{B. Two-dimensional governing equations}

In both fluid media, the formulation is written in terms of pressure. The equation of propagation in the viscous fluid is given by the dissipative wave propagation equation:

$\rho_{f} \ddot{p}=\eta_{f} \Delta \dot{p}+K \Delta p$,

where $p(\boldsymbol{x}, t)$ denotes the pressure field, $\rho_{f}$ mass density, $K$ the fluid compressibility and $\eta_{f}$ the bulk viscosity of the fluid.

In the solid media, the formulation is written in terms of displacement.

Cortical bone is modeled as an heterogeneous material using the linear theory of viscoelastic without memory. The stress tensor $\boldsymbol{\sigma}$ is linearly related to the strain tensor $\boldsymbol{\varepsilon}$ and to the rateof-deformation tensor $\dot{\varepsilon}$ through

$\sigma=C \varepsilon+E \dot{\varepsilon}$

where $C$ is the stiffness tensor and $E$ is the viscoelastic tensor. For a 2-D transverse isotropic medium (in the plane defined by $(x, z))$, the stiffness tensor writes:

$C=\left(\begin{array}{ccc}C_{11} & C_{13} & 0 \\ C_{13} & C_{33} & 0 \\ 0 & 0 & C_{55}\end{array}\right)$,

where all stiffness coefficients are written using the Voigt notation.

The present model is based on data measured for bovine cortical bone, which has been shown to be a significantly anisotropic medium in terms of ultrasonic attenuation as BUA values measured in the axial direction are significantly smaller than BUA values obtained in the radial and tangential directions [9]. Therefore, we have considered an anisotropic (transverse isotropic) tensor $E$ to describe the bone dissipative behavior given by:

$$
E=\left(\begin{array}{ccc}
\eta_{11}(z) & \eta_{13} & 0 \\
\eta_{13} & \eta_{33} & 0 \\
0 & 0 & \eta_{55}
\end{array}\right)
$$

Moreover, in a recent study [10], BUA values were found to depend significantly on bone mineral density as well as on mass density, which are two quantities closely related to the porosity. As the spatial distribution of porosity is heterogeneous in cortical bone [7], the viscoelastic properties of cortical bone are also expected to be spatially dependent.

Therefore, we have considered an heterogeneous behavior of the viscoelastic tensor $E$.

At both interfaces between the fluid layers and the solid layer, the boundary conditions in terms of displacement and normal stresses are taken into account. The internodal distance in both directions is chosen at least smaller than $0.15 \mathrm{~mm}$ in the fluid and $0.25 \mathrm{~mm}$ in the solid, so that it is lower than the smallest wavelength divided by 6 in all directions and both media. 


\section{Determination of a realistic range of variation of material properties}

1) Attenuation coefficient in the soft tissues and bone marrow

We assumed constant values for the mass density $\rho_{f}=1 \mathrm{~g} . \mathrm{cm}^{-3}$ and the compressibility $K=2.2510^{9} \mathrm{~Pa}$ corresponding to an acoustic wave velocity $c_{f}$ of $1500 \mathrm{~m} / \mathrm{s}$ in the absence of viscosity.

Attenuation coefficient data taken from the literature are used in order to define realistic numerical values for the viscosity $\eta_{f}$ of the soft tissues and of bone marrow. Using a model obtained for a system in which a viscous element is placed in parallel with the spring, the attenuation coefficient $\alpha_{f}$ is given by [12]:

$\alpha_{f}=\frac{\eta_{f}}{2 c_{f} K} \omega^{2}$

when the condition $\omega>>\alpha_{f} c_{f}$ is fulfilled.

2) Stiffness tensor of cortical bone

The same approach as the one described in [1] is used in the present work in order to define realistic numerical values for the different components of the stiffness tensor of cortical bone and for their variation.

3) Attenuation coefficient in cortical bone

The determination of realistic values of the components of the viscoelastic tensor $E$ is performed by considering the experimental results of ultrasonic attenuation coefficient measurements obtained in the literature. Similar relationships than Eq. (4) are applied in the case of longitudinal and transverse wave velocities to derive the relation between the attenuation coefficient at $1 \mathrm{MHz}$ (which is obtained from the literature) and $\eta_{11}, \eta_{33}$, and $\eta_{55}$ respectively.

We could not find in the literature a simple way to determine the value of $\eta_{13}$. Therefore, the reference value of $\eta_{13}$ is derived from the mean values of $\eta_{11}$ and $\eta_{55}$ by assuming an isotropic behavior of viscoelasticity. In addition, the minimum and maximum values of $\eta_{13}$ were obtained by verifying that the thermodynamical stability conditions are always respected when varying each material property independently. The physiological ranges of variation of the other viscoelastic constants are obtained by considering the different values of ultrasonic attenuation coefficient measured in the literature.

\section{Modeling a gradient of material property}

The impact of a controlled gradient vector $\boldsymbol{\delta}_{11}$ of $\eta_{11}$ on the FAS velocity is investigated in the case of a relatively thick bone width $(\mathrm{h}=4 \mathrm{~mm})$, all other material properties (stiffness and viscoelastic coefficients) remaining equal to their reference value.

The gradient vector $\boldsymbol{\delta}_{11}=\delta \boldsymbol{e}_{z}$ is assumed to be independent of $x$. Only the simple situation of affine spatial variations of $C_{11}$ is considered, corresponding to a constant value of $\delta$.

The quantity $\eta_{11}(\mathrm{z})$ is given by:

$$
\eta_{11}(\mathrm{z})=\eta_{11 \mathrm{~m}}+\delta z
$$

where $\eta_{11 \mathrm{~m}}$ corresponds to the minimal value of $\eta_{11 \mathrm{~m}}$. The minimal value $\delta_{m}$ of $\delta$ (which corresponds to its maximum in absolute value as $\delta<0)$ is chosen so that $\eta_{11}(-\mathrm{h})$ is equal to $\eta_{11 M}$, where $\eta_{11 M}$ is the maximal value of $\eta_{11}$.

\section{RESULTS}

\section{A. Range of realistic material properties}

The same approach as the one described in [13] is used to derive the attenuation coefficient in bone marrow because experimental measurements could not be found in the literature. Table I shows the different values taken into account for the viscous parameters of bone and of the soft tissues.

TABLE I. MEAN, MAXIMUM AND MINIMUM VALUES OF THE VISCOELASTIC CONSTANTS OF BONE AND SOFT TISSUES

\begin{tabular}{|l|l|l|l|}
\hline & \multicolumn{1}{|c|}{ Mean } & \multicolumn{1}{c|}{ Minimum } & \multicolumn{1}{c|}{ Maximum } \\
\hline$\alpha_{\mathrm{f}}(\mathrm{dB} / \mathrm{cm})$ & 1 & 0 & 2 \\
\hline$\eta_{\mathrm{f}}(\mathrm{Pa} . \mathrm{s})$ & 2 & 0 & 4 \\
\hline$\alpha_{11}(\mathrm{~dB} / \mathrm{cm})$ & 3.2 & 0.8 & 10.6 \\
\hline$\eta_{11}(\mathrm{~Pa} . \mathrm{s})$ & 157 & 39 & 521 \\
\hline$\alpha_{33}(\mathrm{~dB} / \mathrm{cm})$ & 4.2 & 1.7 & 12.8 \\
\hline$\eta_{33}(\mathrm{~Pa} . \mathrm{s})$ & 109 & 44 & 334 \\
\hline$\alpha_{55}(\mathrm{~dB} / \mathrm{cm})$ & 4 & 0 & 8 \\
\hline$\eta_{55}(\mathrm{~Pa} . \mathrm{s})$ & 18 & 0 & 36.2 \\
\hline$\eta_{13}(\mathrm{~Pa} . \mathrm{s})$ & 121 & 39 & 131 \\
\hline
\end{tabular}

B. Dependence of the FAS velocity to changes of viscous and viscoelastic properties of bone and surrounding fluids

From the analysis of numerical simulations, the variation of the FAS velocity due to changes of viscous properties of the soft tissues and of bone marrow within a realistic range was assessed for $\mathrm{h}=0.6$ and $4 \mathrm{~mm}$ and for homogeneous material properties. The FAS velocity is independent of the viscous properties of the soft tissues as well as of marrow within the physiological range. Therefore, the soft tissues as well as bone marrow will be considered as non viscous fluids in what follows.

The variation of the FAS velocity due to changes of the viscoelastic parameters of cortical bone within a realistic range was assessed for $h=4 \mathrm{~mm}$ and for homogeneous material properties. Table II shows that the FAS velocity is only sensitive to variations of $\eta_{11}$.

TABLE II. SENSITIVITY OF THE FAS VELOCITY TO CHANGES OF THE BONE VISCOELASTIC PROPERTIES FOR H=4 MM AND HOMOGENEOUS BONE MATERIAL PROPERTIES. THE COMPUTED FAS VELOCITY IS INDICATED FOR THE MINIMAL AND MAXIMAL VALUES OF EACH VARIABLE CORRESPONDING TO THE REALISTIC RANGE OF VARIATION SHOWN IN TABLE II

\begin{tabular}{|c|c|c|c|c|}
\hline $\begin{array}{c}\text { Material } \\
\text { property (Pa.s) }\end{array}$ & $\boldsymbol{\eta}_{\mathbf{1 1}}$ & $\boldsymbol{\eta}_{\mathbf{1 3}}$ & $\boldsymbol{\eta}_{\mathbf{3 3}}$ & $\boldsymbol{\eta}_{\mathbf{5 5}}$ \\
\hline $\begin{array}{c}\text { FAS velocity for the } \\
\text { minimum value (m/s) }\end{array}$ & 3654 & 3738 & 3732 & 3734 \\
\hline $\begin{array}{c}\text { FAS velocity for the } \\
\text { maximum value (m/s) }\end{array}$ & 3956 & 3735 & 3734 & 3733 \\
\hline
\end{tabular}




\section{Effect of a gradient of bone viscoelastic properties}

Figure 2 shows the dependence of the FAS velocity on $\delta_{11}$ in the case of a thick solid layer $(h=4 \mathrm{~mm})$. The FAS velocity increases when $\delta_{11}$ increases. The dashed line of Fig. 2 shows the FAS velocity obtained when considering a constant homogeneous value of $\eta_{11}$ equal to its spatially averaged values. A poor agreement is obtained between the dashed and solid lines which shows that in the case of a thick bone width with heterogeneous bone properties, the FAS velocity can not be obtained by simply spatially averaging $\eta_{11}$. The difference between the solid and dashed lines may lead to the estimation of a contributing penetration depth using the same method as the one described in [1] (see [11] for more details).

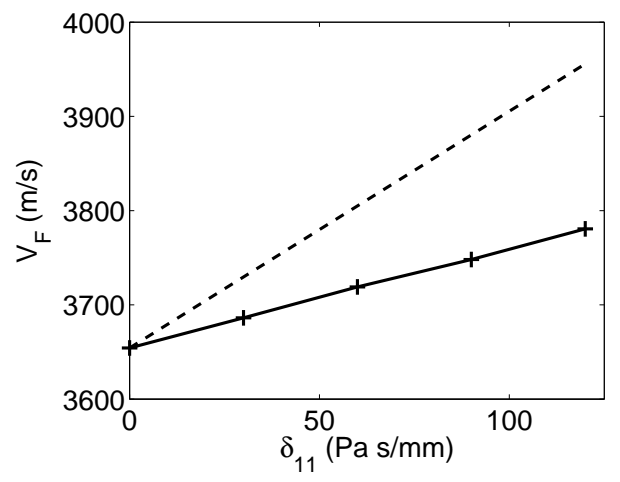

Figure 2. Variation of the FAS velocity versus $\delta_{11}$ for $\mathrm{h}=4 \mathrm{~mm}$. The continuous line indicates the results obtained with the finite element model. The dashed line corresponds to the FAS velocity obtained with homogeneous spatially averaged viscoelastic properties.

\section{DISCUSSION AND CONCLUSION}

To the best of our knowledge, this study is the first one to focus on the effect of ultrasonic attenuation on the FAS velocity estimated with an axial transmission configuration for different solid layer thicknesses. The present study shows that in the studied geometrical configuration, ultrasonic attenuation effects related to the surrounding soft tissues are not likely to modify the results compared to the case with no losses. However, the results shown in Table II suggest that the effect of changes of viscoelastic properties within their estimated physiological range may be of the same order of magnitude as the effect of changes of the stiffness coefficient or mass density within the physiological range [1]. These results indicate that attenuation is an important property which should be accounted for when modeling the ultrasonic propagation in cortical bone in the context of axial transmission. Moreover, the components of the viscoelastic tensor affecting the FAS velocity are the same as the components of the elastic tensor influencing the value of the FAS velocity found in Table II of [1].

\section{ACKNOWLEDGMENT}

This study was supported by the Agence Nationale de la Recherche (Contract BoneChar n BLAN06-2_144779).

\section{REFERENCES}

[1] G. Haiat, S. Naili, Q. Grimal, M. Talmant, C. Desceliers, and C. Soize, "Influence of a gradient of material properties on ultrasonic wave propagation in cortical bone: application to axial transmission," J Acoust Soc Am., vol. 125, pp. 4043-52., 2009.

[2] M. Muller, P. Moilanen, E. Bossy, P. Nicholson, V. Kilappa, J Timonen, M. Talmant, S. Cheng, and P. Laugier, "Comparison of three ultrasonic axial transmission methods for bone assessment," Ultrasound Med Biol., vol. 31, pp. 633-42., 2005.

[3] M. Talmant, S. Kolta, C. Roux, D. Haguenauer, I. Vedel, B. Cassou, E. Bossy, and P. Laugier, "In vivo performance evaluation of bi-directional ultrasonic axial transmission for cortical bone assessment," Ultrasound Med Biol., vol. 35, pp. 912-9. Epub 2009 Feb 25., 2009.

[4] E. Bossy, M. Talmant, and P. Laugier, "Bi-directional axial transmission can improve accuracy and precision of ultrasonic velocity measurement in cortical bone: a validation on test material," IEEE Trans Ultrason Ferroelectr Freq Control., vol. 51, pp. 71-9, 2004.

[5] E. Bossy, M. Talmant, and P. Laugier, "Three-dimensional simulations of ultrasonic axial transmission velocity measurement on cortical bone models," J Acoust Soc Am, vol. 115, pp. 2314-24., 2004.

[6] E. Bossy, M. Talmant, and P. Laugier, "Effect of bone cortical thickness on velocity measurements using ultrasonic axial transmission: a 2D simulation study," J Acoust Soc Am., vol. 112, pp. 297-307., 2002.

[7] V. Bousson, A. Meunier, C. Bergot, E. Vicaut, M. A. Rocha, M. H. Morais, A. M. Laval-Jeantet, and J. D. Laredo, "Distribution of intracortical porosity in human midfemoral cortex by age and gender," J Bone Miner Res., vol. 16, pp. 1308-17., 2001.

[8] C. D. Thomas, S. A. Feik, and J. G. Clement, "Regional variation of intracortical porosity in the midshaft of the human femur: age and sex differences," J Anat., vol. 206, pp. 115-25., 2005.

[9] M. Sasso, G. Haïat, Y. Yamato, S. Naili, and M. Matsukawa, "Frequency dependence of ultrasonic attenuation in bovine cortical bone: an in vitro study," Ultrasound Med Biol., vol. 33, pp. 1933-42, 2007.

[10] M. Sasso, G. Haïat, Y. Yamato, S. Naili, and M. Matsukawa, "Dependence of ultrasonic attenuation on bone mass and microstructure in bovine cortical bone," J. Biomech., vol. 41, pp. 347-55, 2008.

[11] S. Naili, M.-B. Vu, Q. Grimal, M. Talmant, C. Desceliers, C. Soize, and G. Haiat, "Influence of viscoelastic and viscous absorption on ultrasonic wave propagation in cortical bone: application to axial transmission," J Acoust Soc Am., submitted.

[12] D. Royer and E. Dieulesaint, Elastic Waves in Solids I: Free and Guided Propagation. New York, 2000.

[13] G. Haiat, A. Lhemery, F. Renaud, F. Padilla, P. Laugier, and S. Naili, "Velocity dispersion in trabecular bone: influence of multiple scattering and of absorption," J Acoust Soc Am., vol. 124, pp. 4047-58., 2008. 